\title{
The Regular 4-Dimensional 57-Cell
}

\author{
Carlo H. Séquin* and James F. Hamlin \\ CS Division, U.C. Berkeley
}

\section{Motivation}

Interest in regular 4-dimensional polytopes has recently flared up after a brief description of the "11-Cell" in Discover Magazine [Lanier 2007]. But there exists an even more intricate 4-D regular polytope: the "57-Cell." Both objects have high combinatorial symmetry (660 and 3420 automorphisms, resp.), which however can only be observed in much higher-dimensional spaces (10 and 56, resp.). They may be useless objects in 3D space - but perhaps some day they will turn out to be important building blocks in the 10-dimensional space postulated by string theory.

Both the 11-Cell and the 57-Cell are abstract 4-D polychora (multi-cells) bounded by all identical 3-D "surface" cells. Most people in the computer-graphics community are familiar with the 5 completely regular 3-D polyhedra: the Platonic solids. It is also fairly well known that by using these solids as boundary elements, one can construct 6 different, completely regular polychora in 4-D space (the hypercube, and the 5-, 16-, 24-, 120-, and 600-Cell). Most of these objects have been known for more than a century. The 11-Cell [Grünbaum 1976] and the 57-Cell [Coxeter 1982] are much more recent discoveries. They have received very little exposure outside the hardcore mathematics community - mostly because these two objects cannot be represented in 3-D space in any reasonable way. These objects are highly self-intersecting even in 4-D space, because their boundary cells are single-sided manifolds like a Moebius band or a Klein bottle. [Séquin and Lanier 2007] have tried to make the 11-Cell accessible to nonmathematicians. Here we focus on the even more complicated $57-$ cell and try to foster an understanding of this fascinating object. We start by presenting a visualization of the building blocks that comprise the 3-D "surface" or "crust" of this object.
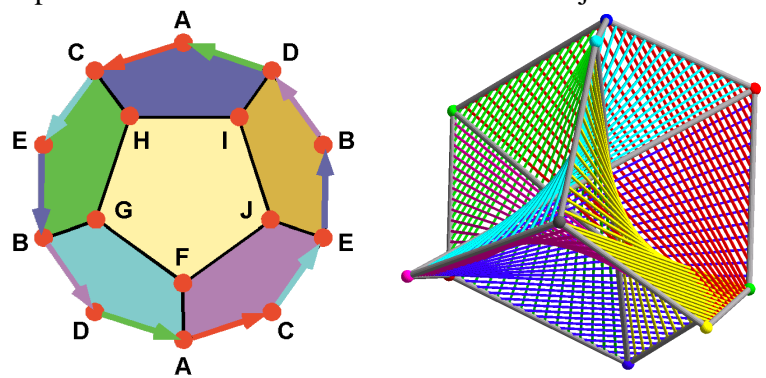

Figure 1. (a) Hemi-dodecahedron, (b) its six warped pentagons.

\section{The Hemi-dodecahedron}

To obtain the building block for the 57-Cell, we cut a regular dodecahedron into two identical halves along its zig-zag equator line. The six pentagons of the "northern" hemisphere are left connected as they are, but in addition we also connect all pairs of opposite points on the equatorial rim; i.e., we glue together the six pairs of equally colored opposite arrows in Figure 1a. This yields an abstract, non-physical object composed of the 10 vertices (A...J) and of 6 pentagons sharing a total of 15 edges. The crosscap connections across the equatorial plane turn this surface into a single-sided 2-manifold with the connectivity of the projective plane. There exist many possible compact models of the projective plane embedded in 3D space: The crosscap, Boy's surface, and Steiner's Roman surface. The latter has the highest degree of symmetry (tetrahedral), and we will thus use its shape to guide us in making a model of the hemi-dodecahedral cell. We arrange the 10 vertices in two concentric clusters with octahedral and tetrahedral symmetry, and then map the graph implied by Figure 1a onto these 10 nodes, so that tetrahedral symmetry is maintained. Optionally, we may distort the position of the six octahedron corners slightly to remove the confusing intersection of the three edges that coincide with the three coordinate axes.

If we trace the periphery of one of the six "pentagonal faces," we note that they are highly warped and wildly intersecting one another. To provide a good visualization of their connectivity and relative positions, we render them as collections of struts that model these interpenetrating surfaces as ruled manifolds, where the diameters and separation of the struts allows us to adjust the faces' "transparency" (Fig.1b).

\section{The Cluster of $\mathbf{5 7}$ Hemi-dodecahedra}

We start the assembly of the 57-Cell by placing one such cell at the origin and surrounding it by 6 more cells, gluing one such cell onto every pentagonal face. We continue this process recursively on all faces - bending our assembly into higher dimensions as necessary to make the required connections. Magically, as we add cell number 57, the 3-D "crust" closes onto itself around a perfectly symmetrical cluster. From the original math literature we know some of the resulting numbers. Overall this cluster has 57 vertices, 171 edges, 171 faces, and 57 hemi-dodecahedral cells, and it is thus self-dual. From this we can readily derive that 5 such cells crowd around every edge, and that 6 edges, 15 faces, and 10 cells converge at every vertex (Fig.2a).
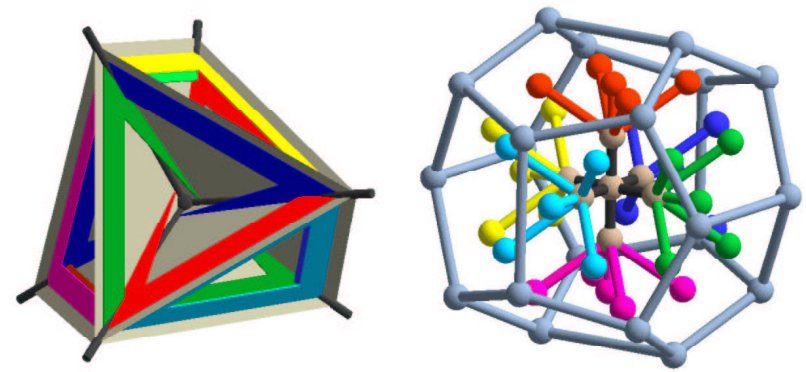

Figure 2. 57-Cell: (a) vertex figure, (b) partial connectivity graph.

To get the "big picture" of the overall composition, it is easier to switch to a dual representation and to study the connectivity graph among the various cells (Fig.2b). Each cell (node) connects to 6 neighbor nodes. No loops of less than 5 edges are allowed. Overall the graph is completely regular and the same as the edge graph. To preserve some of its inherent symmetry, we place the 57 cells on concentric shells with $1,6,30$, and 20 nodes.

\section{References}

COXETER, H.S.M. 1982. Ten toroids and fifty-seven hemi-dodecahedra. Geometriae Dedicata, 13, pp 87-99.

GRÜNBAUM, B. 1977. Regularity of Graphs, Complexes and Designs. Colloque Intern. C.N.R.S., Vol. 260, pp 191-197.

SÉQUIN, C.H. and LANIER, J. 2007. Hyperseeing the Regular Hendecachoron. Proc ISAMA 2007, Texas A\&M, pp 159-166.

LANIER, J. 2007. Jaron's World: Shapes in Other Dimensions. Discover Magazine, April 2007, pp 28-29.

\footnotetext{
*e-mail: sequin@cs.berkeley.edu
} 